\title{
Discussion on the Supervision Mode of Quality and Safety Risk of Consumer Products
}

\author{
Wenjie Chen ${ }^{1}$, Yingcheng $\mathrm{Xu}^{2}$, Wenge $\mathrm{Liu}^{1}$, Xiuli Ning ${ }^{2}$, Teng $\mathrm{Xu}^{3}$, Qingyun $\mathrm{GaO}^{1}$ and $\mathrm{Ning}^{\mathrm{Li}} \mathrm{i}^{1}$ \\ ${ }^{1}$ Product Quality Inspection Institute of Inner Mongolia Autonomous Region, Inner Mongolia 010070, China \\ ${ }^{2}$ Quality Management Branch, National Institute of Standardization, Beijing 100191, China \\ ${ }^{3}$ Food Inspection and Testing Center of Inner Mongolia Autonomous Region, Inner Mongolia 010010, China
}

\begin{abstract}
In recent years, China, as the world's largest consumer products manufacturing country, has more and more consumer quality and safety incidents. By analyzing and constructing the supervision mode of quality and safety risks of consumer products, this paper improves the identification and collection system of quality and safety risks of consumer products, improves the evaluation system of impact factors of quality and safety of consumer products, and strengthens the communication and operation mechanism of quality and safety risks of consumer products. Through the supervision of quality and safety risks of consumer products, we will explore the establishment of a social governance model of quality and safety of consumer products, and build a "five-in-one" supervision model of quality and safety risks of consumer products based on government supervision, enterprise self-discipline, social coordination, public participation and legal guarantee.
\end{abstract}

Keywords: Consumer products; Risk supervision; Risk communication.

\section{The situation of domestic consumer products quality and safety}

At present, as the world's largest consumer products manufacturing country, China's consumer products quality and safety has not only affected the domestic consumers' livelihood issues, but also affected the international competitiveness of China's consumer products manufacturing industry. With the increase in the volume and variety of consumer products in China, the risk of exposure is increasing. According to the statistics of the European Union Non-Food Consumer Products Rapid Alert System (RAPEX) and the China National Institute of Standardization National Consumer Products Safety Internet Public Opinion Monitoring System (CNIS), From January 2004 to July 2018, RAPEX has released more than 7,800 recalls of consumer products made in China, involving smart home appliances, children's products, lamps and other consumer products. Therefore, the quality improvement of consumer products urgently needs to change the mode of supervision, from "consumer products inspection" to "safety risk supervision". Supervision of quality and safety risk of consumer products is an effective means to reduce quality defects and improve quality of consumer products. From the perspective of quality and safety of consumer products, this paper discusses the supervision mode of quality and safety of consumer products and establishes the supervision system of quality and safety of consumer products according to the general steps of risk assessment of consumer products ${ }^{[1]}$. It plays an important role in improving the quality of consumer products and promoting the safety management of consumer products in China.

At present, at the national level, the AQSIQ Defective Product Administrative Center (DPAC) and the AQSIQ Product Quality and Safety Risk Monitoring Center and other international centers have studied and controlled the risk safety of various consumer products. The AQSIQ Product Administrative Center has established the National Injury SurveillaIlce System (NISS) and the AQSIQ Consumer Products Quality and Safety Risk Monitoring Center has established the National Consumer Products Safety Risk Monitoring System and the Public Opinion Monitoring System (CNIS). Through these platforms, it is possible to collect consumer products safety risk information in a timely and effective manner.

However, China has not yet formed a good social atmosphere and consumption habits on the supervision of consumer products safety risks. As a result, the consumer products which may cause casualties and property losses can not be effectively dealt with. Generally speaking, it is difficult to collect the safety risks of consumer products in China. The collection and application of information is still in its infancy, and the society has not formed a good supervision atmosphere. This is also the focus of 
government departments needing more publicity and guidance. With the automobile recall as a reference, it takes 10 years to implement the automobile recall to form a relatively perfect system and a good social atmosphere. In the recall information monitoring, it is very difficult to obtain information about defective consumer products. In July 2016, the American Consumer Product Safety Commission announced a large-scale recall of electric balancing vehicles involving more than 500,000 balancing vehicles of nearly 10 brands. All the recalled electric balancing vehicles are all originated in China, which can be regarded as a very serious recall. Whether the products are sold domestically or not, and whether they are exported abroad and then re-sold domestically, need to be investigated. The Shanghai Monitoring Sub-center monitors the recall incident at the first time. But in collecting consumer products exporters' information, only one of the domestic suppliers of one brand of chargers is found in more than 10 models of electric balancing vehicles, and the rest of the information is unavailable. The fragmentation and disintegration of information investigation is very serious.

\section{Supervision mode of quality and safety of consumer products}

\subsection{Risk identification and collection}

At present, there are many ways to identify and collect the quality and safety risks of consumer products in China, such as product quality and safety risk monitoring, injury monitoring, supervision and spot check, notification and recall, quality arbitration and consumer complaints. Risk monitoring is to discover regional, industrial and systematic quality and safety risks through dynamic collection and analysis of risk information, and to put forward predictive suggestions and countermeasures to prevent the occurrence or spread of risk from becoming a serious quality and safety event. Injury monitoring refers to the long-term and continuous collection of data on the occurrence, death, disability and economic loss of different groups of people, and clarifies the characteristics and trends of the type of injury and the time distribution of the population. It is used for the optimal injury prevention and control methods related to the population and the environment, the population and the cost-effectiveness, and evaluates the effect of the injury control work to achieve the purpose of reducing the injury event. Consumer products quality and safety monitoring information sources include quality supervision, hospitals, fire protection, associations, sellers, consumers, media, communities, etc. By collecting relevant information of these stakeholders, a database for identifying and collecting consumer products quality and safety risks is established. Specific consumer products quality and safety risk information identification and collection system is shown in Fig. 1.

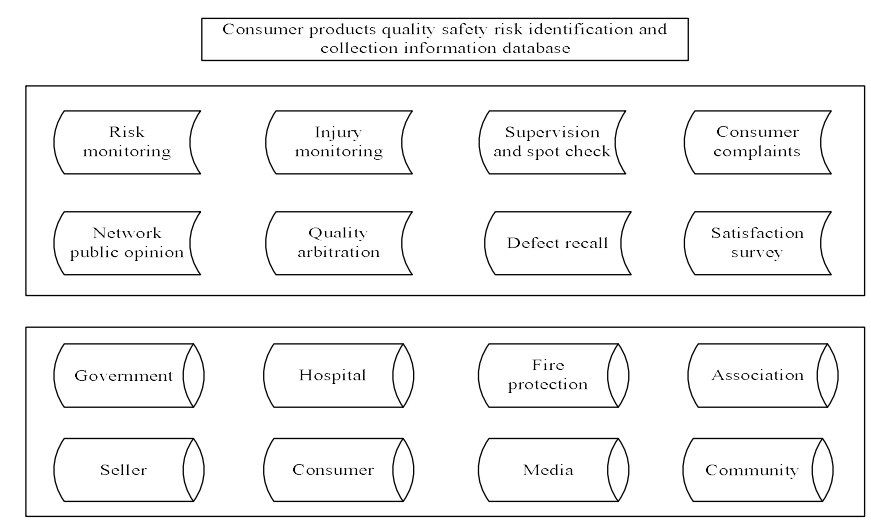

Figure 1. Consumer quality safety risk information identification and collection database.

\subsection{Risk analysis and assessment}

After the comprehensive and in-depth collection of consumer quality and safety risk information, the risk in the database is assessed scientifically. Risk assessment is relatively late in consumer products quality and safety. The study is initially carried out to assess the quality safety risk of agricultural products. The quality and safety factors of consumer products are divided into biological factors, physical factors and chemical factors according to their nature. There are mainly two kinds of models for the quality and safety risk assessment of consumer products: One is the quality and safety factor assessment model based on the risk priority number, and the other is the quality and safety factor assessment model based on the risk matrix. These models can effectively identify the impact factors, as shown in Fig. 2. In a given scenario, the risk level of quality and safety factors is assessed under the joint action of human factor, material factor and environment. ${ }^{[2][3]}$ Sun Jiatian and others introduce the concept of quality and safety impact factors of consumer products, and analyze the relationship between quality and safety impact factors and quality and safety risks. According to the general risk assessment method, the idea and research steps of quantifying the quality and safety risk of consumer products with impact factors are put forward. ${ }^{[4]}$ Cai Huali and others establish the assessment system of quality and safety impact factors of consumer products, construct the automatic safety impact factor assessment system by artificial intelligence, and effectively and quickly realize the assessment of quality and safety impact factors of consumer products based on expert knowledge. ${ }^{[5]}$

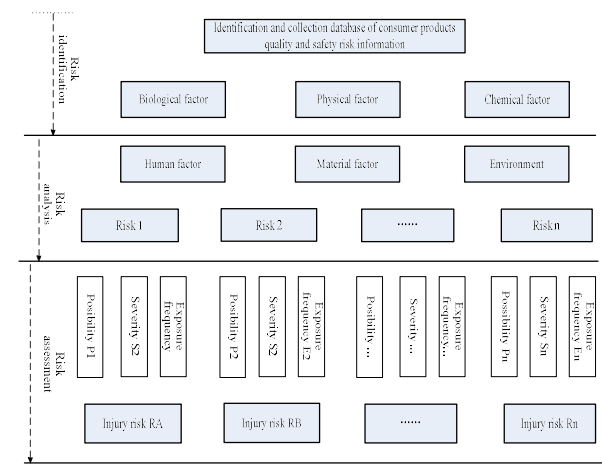

Figure 2. Analysis and assessment of consumer quality and safety risk information. 


\subsection{Risk management and communication}

Risk management is the way to formulate and implement risk control plans, determine the possibility of reducing risk occurrence and reduce its adverse effects. Risk management mainly includes avoiding risks, reducing risks, transferring risks and accepting risks. In the disposal of consumer products quality and safety risks, it is mainly to avoid risks and reduce risks. The current effective methods include defective product recall, defective product destruction, enterprise interview self-examination and other disposal methods. In 1989, the U.S. National Research Council's Risk Awareness and Communication Committee introduced the concept of risk communication in Improving Risk Communication. Risk communication can be used as a risk management method, which can effectively reduce and avoid risks in the implementation process. Risk communication is an interactive process of exchanging information and opinions among individuals, groups and institutions. [6] Risk communication is to link multiple stakeholders involved in the process of risk supervision. Successful communication of consumer products quality and safety risks requires information sharing, exchange and communication between governments, experts, enterprises and consumers and stakeholders. A consensus is reached on consumer products quality and safety information, to fill the "vacuum" between the government and consumers on injury risks, to encourage enterprises to actively release consumer products safety information, and to actively guide social public opinion and consumer sentiment.

China's risk communication research is relatively late, and currently it is mainly about the communication research of security risks in the field of food and agricultural products. According to the safety risk communication project of Japan introduced by Zhang Wensheng and others, its main core idea is to realize the transparency of product information through the construction of information communication between enterprises and consumers. ${ }^{[7]}$ The communication of quality and safety risks for consumer products promotes the collaboration between consumer products enterprises, service institutions, consumers and other private subjects and government departments. By consciously carrying out "communication key points" activities, enterprises can increase the participants of the activities, expand the scope of communication, and improve consumers' trust in consumer products enterprises. Combining with the operation mechanism of the food communication project in Japan ${ }^{[8]}$, and based on the quality and safety of China's consumer products, the communication operation mechanism of China's consumer products quality and safety risks is constructed as shown in Fig. 3 .

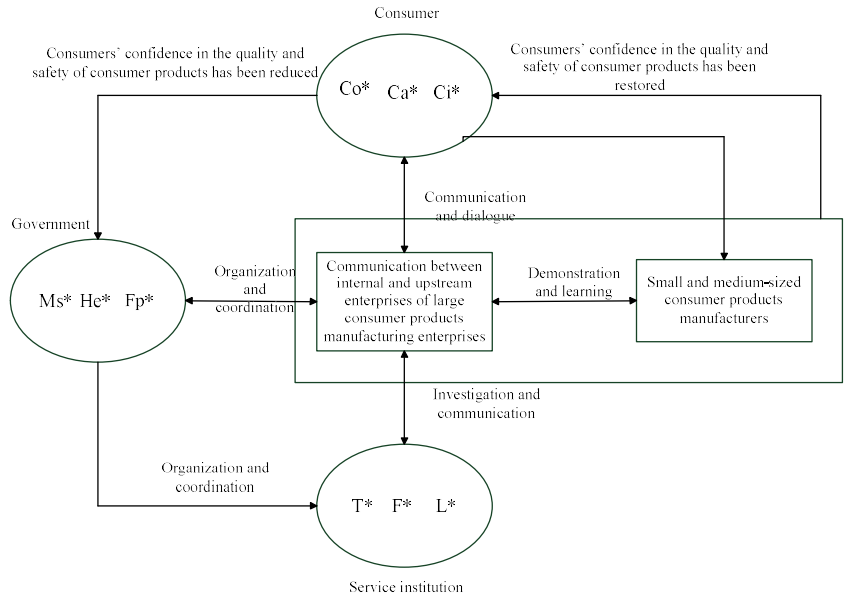

*Co: Consumer; Ca: Consumer association; Ci: Consumer institution; Ms: Market supervision; He: Health and epidemic prevention; Fp: Fire fighting and public security; T: Testing institution; F: Financial institution; L: Legal institution

Figure 3. Operation mechanism of quality and safety risk communication of consumer products.

\section{Prospects for quality and safety risk supervision of consumer products}

Government departments should further strengthen the collaboration between consumer products safety risk supervision departments on the basis of current work. Outside the system, a normalized cooperation mechanism should be formed with relevant departments such as health and epidemic prevention, commerce commission, fire fighting and public security departments to track and analyze the production of consumer products (commerce commission), domestic sales (industry and commerce bureau), injury (hospital, fire fighting, etc.). If special commodities are involved, other relevant departments should intervene to form a complete closed-loop consumer products risk collection survey. It is conducive to collecting effective information, integrating social resources, exploring various channels to carry out consumer products safety risk supervision, and improving the supervision process of consumer products safety risk.

We should strengthen the use of monitoring information, in-depth study the assessment system of consumer products quality and safety impact factor and actively carry out application, timely analyze and verify the consumer products injury information. In order to reduce and eliminate the quality and safety risks of consumer products, we should issue early warning and implement recall to the consumer products which have been proved to have quality and safety defects, tamp the basis of quality and safety risk supervision, and put forward suggestions for the corresponding policies of government departments.

We should clarify the quality and safety risk communication role of consumer products, and form a three-dimensional prevention and control pattern for the prevention and control of consumer products quality and safety risks. From the perspective of the government, production enterprises, sales enterprises, service institutions and consumers and related associations, we should give full 
play to the role of multiple subjects, attach importance to the supervision of the source of consumer products, and emphasize the leading role of enterprises in the safety of consumer products. We will actively encourage and guide enterprises involved in risk commination for the quality and safety of consumer products, explore ways to establish a social governance mode for the quality and safety of consumer products, and build a "five-in-one" mode for risk supervision for the quality and safety of consumer products through government regulation, enterprise self-discipline, social coordination, public participation and legal protection.

\section{Acknowledgments}

We would like to acknowledge that this research are supported and funded by the National Science Foundation of China under Grant No. 91646122 and 91746202, the Basic Scientific Research Business Projects 552018Y-5927, the National Key R\&D Program of China under Grant No.2016YFF0202600 and No.2016YFF0202604.

\section{References}

1. Liu Xia, Luo Hongqi. Product Safety and Risk Assessment [J]. China Association for Quality, 2009 (9): 10-11.
2. Xie Qiuhui, Wei Biwen, $\mathrm{Yu}$ Wenjia, etc. The establishment of risk assessment trend of exported light and textile consuming products and discussion [J]. Shanghai Textile Science \& Technology, 2010, 38 (5): 61-62.

3. Xie Qiuhui, Lu Weimin, Wei Mengyuan, etc. Risk Assessment for Consumer Products of Light and Textile Industry [J]. Shanghai Textile Science \& Technology, 2010, 38 (6): 57-59.

4. Sun Jiatian, Zhang Zihan, Qin Yang. Quantitative Risk Evaluation Based on Impact Factors of Quality Safety [J]. Standard Science, 2010, 422 (4): 4-8.

5. Cai Huali, Yang Yuexiang, Liu Xia, etc. System Design and Implementation for Impact Factors for Consumer Product Quality and Safety [J]. Technique and method, 2012, 12 (4): 53-56.

6. US National Research Council, Improving Risk Communication Washington, D.C: National Academy Press, 1989, p.21.

7. Zhang Wensheng, Wang Shuo, An Yufa, et al. Research on the System Structure and Operation Mechanism of Food Communication Project in Japan [J]. Problems of Agricultural Economy, 2017, 1: 100-108.

8. Liu Chang, An Yufa, Nakajima Kangbo. A Study on the Operation Mechanism and Function of Food Communication Project in Japanese Food Industry. Journal of Public Management, 2011 (4): 96-102. 